\title{
Persönlichkeitsanalysetools - Entscheidungshilfe durch Persönlichkeitstests?
}

\section{Entscheidungshilfen mithilfe psychologischer Eignungstests?}

\section{Allgemeines}

Der Begriff „Persönlichkeitsanalysetool“ ist zunächst einmal lediglich der Oberbegriff für eine Vielzahl von im Internet zu findenden Angeboten. ${ }^{1}$ Mit Hilfe eines vorgegebenen Verfahrens soll das Verhalten und die Persönlichkeit einer Person möglichst objektiv festgestellt werden. ${ }^{2}$ Dabei soll es vor allem darauf ankommen, dass nach Möglichkeit ein vielschichtiges und genaues Bild einer Persönlichkeit erlangt wird, indem auch unbewusste Verhaltensweisen oder/und Einstellungen erfasst werden. ${ }^{3}$ Während solche Analysen aus Zeitschriften, entsprechender Ratgeberliteratur zur Persönlichkeitsentwicklung und der Psychotherapie zumeist bekannt sein bzw. vom einen oder anderen Leser auch schon einmal gelöst worden sein dürften, haben Persönlichkeitsanalysetools aus datenschutzrechtlicher Sicht insbesondere im Zusammenhang mit Beschäftigungsverhältnissen Bedeutung erlangt. Zunächst vorwiegend im Rahmen des Auswahlverfahrens von Bewerbern (hauptsächlich in sog. Assessment-Centern) eingesetzt, sollten diese Tests dazu dienen, die Eignung einer Person für einen bestimmten Arbeitsplatz festzustellen. Der Einsatz im Arbeitsleben beschränkt sich aber schon lange nicht mehr auf die „Begründung eines Beschäftigungsverhältnisses“ (Einstellungsverfahren), sondern erstreckt sich zunehmend auch auf bestehende Arbeitsverhältnisse, um dort beispielsweise vorhandenen Fortbildungsbedarf, die Eignung für eine Beförderung oder die Teamfähigkeit festzustellen und bei Defiziten reagieren zu können. Die sich aus einem Test ergebenden Aussagen können vielfältig sein und zum Beispiel sowohl Verhaltensweisen, Einstellungen, Überzeugungen, Wertvorstellungen, Vorlieben, Stärken, Schwächen als auch alle sonstigen Charaktereigenschaften des Mitarbeiters erfassen.

\section{Arten}

Für die Durchführung von Persönlichkeitstests gibt es ganz unterschiedliche Ansätze. Zum einen besteht die Möglichkeit, dass die zu beurteilende Person Fragen beantwortet (vorgegebene Antworten ankreuzt) und so das am meisten bzw. am wenigsten Zutreffende markiert, was dann mit den durchschnittli-

1 Um die vielen Angebote mit entsprechenden Links zu erhalten, muss lediglich der Begriff "Persönlichkeitsanalysetool“ in ein Suchprogramm eingegeben werden; es finden sich dann sogar astrologische Persönlichkeitsanalysen für Kinder, dazu bspw. unter http://www.cortesi.ch/analysen/an_kindform.htm (letzter Abruf: 1.2.2016), oder Tests, durch die Arbeitsbeziehungen analysiert werden können, vgl. bspw. unter http://www.cortesi.ch/analysen/an_bezform.htm (letzter Abruf: 1.2.2016)

2 Vgl. bspw. unter http://www.zeitzuleben.de/psyche-auf-dem-prufstandpersonlichkeitstests/2/ (letzter Abruf: 2.1.2016).

3 Siehe http://www.zeitzuleben.de/psyche-auf-dem-prufstand-personlichkeitstests/2/ (letzter Abruf: 2.1.2016). chen Werten einer Normstichprobe verglichen wird. ${ }^{4}$ Eine andere Form der Analyse kann in der Auswertung von Materialien, die durch die Testperson selbst hergestellt oder dieser zur Beurteilung vorgelegt wurden, bestehen. ${ }^{5}$ Insgesamt geht es bei der Analyse der Mitarbeiterpersönlichkeiten darum, Verhaltensdimensionen, aktuelle Stimmungszustände, Interessensfelder, Persönlichkeitsmerkmale und Motivationsausprägung zu erfassen. ${ }^{6}$ Je nach angewendetem Verfahren werden die Testpersonen dann entsprechend den auf die gestellten Fragen gegebenen Antworten unterschiedlichen Persönlichkeitsmustern (Typenbeschreibungen) zugeordnet oder die einzelnen Eigenschaften des Betroffenen beschrieben und deren Ausprägung anhand von Skalen dargestellt. ${ }^{7}$

\section{Problem}

Die Schwierigkeit des Einsatzes derartiger Persönlichkeitsanalysen liegen dabei nicht allein in der richtigen Wahl eines wissenschaftlich fundierten Auswertungssystems. ${ }^{8} \mathrm{Zu}$ berücksichtigen ist vielmehr, dass es sich sowohl bei den gegebenen Antworten als auch bei deren Auswertungen bzw. den Ergebnissen um personenbezogene Daten im Sinne des Bundesdatenschutzgesetzes handelt, die je nach Ausrichtung auch besondere Arten personenbezogener Daten im Sinne des $₫ 3$ Abs. 9 BDSG betreffen können. ${ }^{9}$ Deren Verarbeitung bedarf demzufolge einer Rechtsgrundlage (vgl. $\$ 4$ Abs. 1 BDSG) bzw. ist im Einzelfall sogar besonderen Restriktionen unterworfen. ${ }^{10}$ Neben den sich daraus ergebenden zahlreichen datenschutzrechtlichen Fragen, ${ }^{11}$ tritt gerade im Arbeitsleben das Problem hinzu, dass die (Fehl-) Einschätzung durch ein unzureichendes Persönlichkeitsanalysetool durchaus weitreichende Folgen für Betroffene haben kann, hängt davon doch die Begründung bzw. Weiterführung einer Beschäftigung oder auch das Vorankommen im Unternehmen ab.

4 Vgl. unter anderem unter https://de.wikipedia.org/wiki/Pers\%C3\%B6nlichkeitstest (letzter Abruf: 2.2.2016).

5 Vgl. https://de.wikipedia.org/wiki/Pers\%C3\%B6nlichkeitstest oder http:// www.zeitzuleben.de/psyche-auf-dem-prufstand-personlichkeitstests/2/ (letzter Abruf: 2.2.2016).

6 Dazu näher bei Bausewein, DuD 2016/3, S. 139 in diesem Heft.

7 Zum Ganzen mit weiteren Nachweisen Bausewein, DuD 2016/3, S. 139 in diesem Heft.

8 Zu den Auswahlkriterien u. a. näher bei Hornke/Kersting, Stellungnahme zum Gutachten von Eckstaller u. a., S. 2, abrufbar unter http://www.bdp-verband. org/bdp/politik/2005/50819_insights.pdf (letzter Abruf: 3.1.2016) oder auch Müllerschön, Persönlichkeitstests auf dem Prüfstand, S. 1 ff., abrufbar unter http:// www.muellerschoen-beratung.de/fileadmin/pdf/PersoenlichkeitstestsPruefstand.pdf (letzter Abruf: 3.1.2016).

9 Zum Personenbezug der Daten siehe auch bei Bausewein, DuD 2016/3, S. 139 in diesem Heft.

10 Allgemein dazu Gola/Schomerus, BDSG, § 3 Rn. $56 \mathrm{ff}$.

11 Bspw. zur Auftragsdatenverarbeitung siehe Eckhardt/Kramer, DuD 2016/3, S. 144 in diesem Heft. 